\title{
Observed Steady State Volume of Distribution Normalized by Weight
}

National Cancer Institute

\section{Source}

National Cancer Institute. Observed Steady State Volume of Distribution Normalized by

Weight. NCl Thesaurus. Code C102380.

The volume of distribution at steady state based on the observed CLST for a substance administered by intravascular dosing divided by the weight. 